\title{
Fatores associados a metas de utilização de serviços em municípios da Bahia com
} Centros de Especialidades Odontológicas, de 2013 a 2019

\author{
Associated factors with service utilization goals in municipalities of Bahia with Dental Specialty
}

Centers from 2013-2019

Factores asociados a las metas de utilización de servicios en los municipios de Bahía con Centros de Especialidades Odontológicas, de 2013 a 2019

\section{Resumo}

Objetivo: Identificar os fatores associados ao cumprimento de meta de utilização dos serviços odontológicos na atenção básica e atenção especializada dos municípios com CEO do estado da Bahia, de 2013 a 2019. Metodologia: Este estudo é do tipo transversal, exploratório e analítico, utilizando dados secundários obtidos do Sistema de Informação Ambulatorial, da plataforma WEB e-Gestor Atenção Básica, do Sistema de Informações sobre Orçamentos Públicos em Saúde e do $1^{\circ}$ ciclo do Programa Nacional de Melhoria do Acesso e da Qualidade dos Centros de Especialidades Odontológicas. Foram analisados 73 municípios da Bahia com equipe de saúde bucal implantada e com CEO, através dos indicadores de utilização propostos para saúde bucal pelo Ministério da Saúde. As variáveis foram relacionadas às características demográficas, financiamento e oferta dos serviços, sendo a variável resposta o cumprimento de $50 \%$ de dois padrões da Portaria $\mathrm{N}^{\circ} 1.631$ de 1 de outubro de 2015. Resultados: Na amostra final, 58,90\% dos municípios eram de pequeno porte, 50,68\% mostraram acima de 85,9\% de participação das transferências intergovernamentais na receita total, 49,32\% tinham cobertura de saúde bucal na atenção básica acima de 77,7\% e 43,83\% possuíam CEO tipo I. Houve significância estatística na associação entre cobertura de saúde bucal e cumprimento de $50 \%$ ou mais da meta. Conclusão: Foi evidenciado que municípios de menor porte, com participação das transferências intergovernamentais na receita total acima de $85,9 \%$, com cobertura de saúde bucal acima de $77 \%$ e com CEO tipo I, obtiveram maior cumprimento de meta. Conclui-se que a baixa cobertura de saúde bucal contribui para o não cumprimento de $50 \%$ da meta.

Palavras-chave: Saúde bucal; Políticas de saúde; Serviços de saúde; Financiamento da saúde.

\begin{abstract}
Objective: To identify the factors associated with the achievement of the goal of utilization of dental services in primary care and specialized care of the municipalities with a CEO in the state of Bahia, from 2013 to 2019. Methodology: This is a cross-sectional, exploratory and analytical study, using secondary data obtained from the Outpatient Information System, the WEB platform e-Gestor Basic Care, the Information System on Public Health Budgets and the 1st cycle of the National Program for Improving Access and Quality of Dental Specialty Centers. We analyzed 73 municipalities in Bahia with an implemented oral health team and with the CEO, through the utilization
\end{abstract}


indicators proposed for oral health by the Ministry of Health. The variables were related to demographic characteristics, financing and supply of services, and the response variable was compliance with 50\% of two standards of the ordinance $\mathrm{N}^{\mathrm{o}} 1.631$ de 1 de october de 2015 . Results: In the final sample, $58.90 \%$ of the municipalities were small, $50.68 \%$ showed above $85.9 \%$ participation of intergovernmental transfers in total revenue, $49.32 \%$ had oral health coverage in primary care above $77.7 \%$ and $43.83 \%$ had CEO type I. There was statistical significance in the association between oral health coverage and achievement of $50 \%$ or more of the goal. Conclusion: It was evidenced that smaller municipalities, with participation of intergovernmental transfers in total revenues above $85.9 \%$, with oral health coverage above $77 \%$ and with a type I CEO, achieved greater compliance with the goal. It is concluded that low oral health coverage contributes to the noncompliance of $50 \%$ of the goal.

Keywords: Oral health; Health policies; Health services; Health financing.

\begin{abstract}
Resumen
Objetivo: Identificar los factores asociados al logro de la meta de utilización de los servicios odontológicos en los atención primaria y atención especializada de los municipios con CEO en el estado de Bahía, de 2013 a 2019. Metodología: Se trata de un estudio transversal, exploratorio y analítico, a partir de datos secundarios obtenidos del Sistema de Información de Consultas Externas, la plataforma WEB e-Gestor Basic Care, el Sistema de Información de Presupuestos de Salud Pública y el 1er ciclo del Programa Nacional de Mejora del Acceso y la Calidad de los Centros de Especialidades Odontológicas. Se analizaron 73 municipios de Bahía con equipo de salud bucal implantado y con CEO, a través de los indicadores de utilización propuestos para la salud bucal por el Ministerio de Salud. Las variables estaban relacionadas con las características demográficas, la financiación y la oferta de servicios, y la variable de respuesta era el cumplimiento del $50 \%$ de dos normas de la ordenanza $\mathrm{N}^{\mathrm{o}} 1.631$ de 1 de octubre de 2015. Resultados: En la muestra final, el 58,90\% de los municipios eran de pequeño tamaño, el 50,68\% presentaban una participación de las transferencias intergubernamentales en los ingresos totales superior al 85,9\%, el 49,32\% tenían una cobertura sanitaria en atención primaria superior al 77,7\% y el 43,83\% tenían un CEO de tipo I. Hubo significación estadística en la asociación entre la cobertura de salud bucal y el cumplimiento del $50 \%$ o más de la meta. Conclusión: Se demostró que los municipios de menor porte, con participación de las transferencias intergubernamentales en la recepción total por encima del 85,9\%, con cobertura de salud bucal por encima del $77 \%$ y con CEO tipo I, obtuvieron un mayor cumplimiento de la meta. Se concluye que la baja cobertura de salud bucal contribuye a que no se cumpla el 50\% de la meta.
\end{abstract}

Palabras clave: Salud bucodental; Políticas sanitárias; Servicios sanitários; Financiación sanitaria.

\title{
1. Introdução
}

O Sistema Único de Saúde (SUS) consolidou-se a partir da década de 90, após a promulgação da Lei № 8.080/90. No ano de 1994, foi criado o Programa de Saúde da Família (PSF). O PSF inicia o debate para uma estratégia prioritária de organização e fortalecimento da atenção básica, a Estratégia de Saúde da Família (ESF). A equipe de saúde bucal (ESB) foi incorporada no ano 2000, possibilitando uma nova forma de planejamento e programação na atenção básica (AB) (Pimentel et al., 2012; Pereira, 2013).

No ano de 2004, o Ministério da Saúde (MS) lançou a Política Nacional de Saúde Bucal (PNSB), Programa Brasil Sorridente, que tem como objetivo garantir as ações de promoção, prevenção e recuperação da saúde bucal (SB) dos brasileiros. A PNSB também incorporou o acesso à atenção especializada (AE) através dos Centros de Especialidades Odontológicas (CEO) e Laboratórios Regionais de Próteses Dentárias (LRPD) (Brasil, 2004; Chaves, Cruz, Barros, \& Figueiredo, 2011). No Brasil, no ano de 2019 foram implantados 14 CEO representando um total de 1.175 e 2.703 LRPD (MS, 2019; OAPS, 2021). Com relação à atenção básica, a cobertura de saúde bucal em 2019 representou 52,97\% com 28.991 equipes de saúde bucal na ESF (MS, 2019).

Os CEO podem se apresentar em três categorias distintas: CEO tipo I (com três cadeiras odontológicas), tipo II (com quatro a seis cadeiras odontológicas) e tipo III (com sete ou mais cadeiras odontológicas). Todos os CEO devem ofertar minimamente as cinco especialidades mínimas: diagnóstico bucal, com ênfase no diagnóstico e detecção do câncer bucal, periodontia especializada, cirurgia oral menor, endodontia e atendimento a portadores de necessidades especiais, e todas as cadeiras devem funcionar por 40 horas semanais (Machado, Silva, \& Ferreira, 2015; Gonçalves et al., 2018). 
Alguns autores apontaram a necessidade de estudos para monitorar e avaliar os impactos da política. Barros, Cangussu, Cruz, Silva e Roncalli (2016) relataram a importância de investigações que possibilitem a análise de indicadores de saúde e saúde bucal articulada com fatores sociais, políticos e históricos na implantação da atenção à saúde bucal a fim de elucidar as diferenças existentes nos municípios. Existem evidências de que a inclusão do dentista na ESF melhorou a satisfação do usuário, aumentou acesso e utilização nas áreas mais necessitadas e cobertas, mas não há um consenso sobre grandes transformações nas práticas e na utilização dos serviços (Rocha \& Goes, 2008; Pereira et al., 2012; Mattos, Ferreira, Leite, \& Greco, 2014).

Neves, Giordani e Hugo (2019), trouxeram a importância de entender as características demográficas, os indicadores do sistema de saúde dos municípios brasileiros, equipamentos, insumos e características do processo de trabalho, sob a ótica conceitual da integralidade, pois mesmo com mais de 10 anos da ESB na ESF os achados ainda são insatisfatórios. Baldani, Ribeiro, Gonçalves, e Ditterich (2018), relataram a importância da gestão como apoiadora do processo de trabalho das equipes, os achados evidenciam desigualdades intermunicipais, desfavoráveis aos municípios menores e mais carentes. É necessária a qualificação da gestão a fim de favorecer o resgate de competências e habilidades indispensáveis para a boa gestão da clínica e do cuidado na atenção básica.

Os estudos realizados mostraram a relação entre porte populacional e utilização de serviços, aumento da oferta de serviços odontológicos ao longo dos anos no Brasil, desigualdades na utilização, apontando um menor acesso para os usuários com maior vulnerabilidade social (Rocha \& Goes, 2008; Pereira et al., 2012; Mattos et al., 2014).

Apesar dos avanços com a PNSB e o crescimento dos serviços odontológicos, ainda há um subfinanciamento dos serviços de saúde. Ainda que os municípios recebam os recursos federais, estes não conseguem garantir a oferta dos serviços de saúde bucal, existem gastos adicionais com manutenção de equipamentos, instrumentais, insumos e materiais de consumo específicos que são de alto custo (Kornis, Maia, \& Fortuna, 2011).

Os estudos que se debruçam sobre o financiamento abordam os repasses e gastos com a saúde bucal (Kornis et al., 2011; Rossi, Chaves, Almeida, Santos, \& Santana, 2018). Aragão, Amorim, e Conceição (2019) estudaram o financiamento em dois municípios da Bahia, Feira de Santana e Vitória da Conquista, no ano de 2017. Com relação à saúde bucal na AB, Feira de Santana investiu do gasto total 2,5\% e Vitória da Conquista 4,6\%, já na média e alta complexidade investiu 0,16\% e $0,26 \%$, respectivamente, estes baixos investimentos refletem na baixa cobertura dos serviços odontológicos. Rossi et al. (2018), em estudo dos anos de 2003 a 2017 sobre o financiamento da política nacional de saúde bucal do Brasil, relataram que até o ano de 2016 houve uma constância no repasse para AB, tendo decréscimo em 2017. Na AE, houve aumento da participação principalmente a partir de 2013, demonstrando que não há uma pactuação entre os níveis de complexidade, fragilizando, assim, a integralidade pretendida.

Ademais, não foram identificados estudos que analisem os fatores associados, no âmbito municipal, à utilização dos serviços odontológicos da atenção básica e atenção especializada da Bahia, no que se refere ao cumprimento de metas. Nesse sentido, esse trabalho buscou identificar os fatores associados ao cumprimento de meta de utilização dos serviços odontológicos na AB e AE dos municípios com CEO do estado da Bahia, de 2013 a 2019.

\section{Metodologia}

Este estudo foi do tipo transversal exploratório e analítico, utilizando dados secundários obtidos da plataforma WEB e-Gestor Atenção Básica (AB), do sistema de informação ambulatorial SIA/SUS, do $1^{\circ}$ ciclo do Programa Nacional de Melhoria do Acesso e da Qualidade dos Centros de Especialidades odontológicas (PMAQ-CEO), do site do Instituto Brasileiro de Geografia e Estatística (IBGE) e do Sistema de Informações sobre Orçamentos Públicos em Saúde (SIOPS). 
A Bahia, estado da região Nordeste, possui uma população estimada de 14.930 .634 (2020), apresentando Índice de Desenvolvimento Humano (IDH) médio - 0,660, sendo o IDH do Brasil 0,699 (IBGE, 2010). Representa o maior estado da região Nordeste em área e população, com grande quantidade de ESB e CEO em relação aos demais estados da região (MS, 2019). Foram analisados os dois últimos períodos de governo municipal, 2013-2016 e 2017-2019. No que se refere ao ano de 2020, seriam analisados apenas os meses do primeiro trimestre, mas por conta da pandemia do novo coronavírus-Sars-Cov-2 (Covid-19), este ano foi desconsiderado.

Para a realização deste estudo, foram considerados 73 municípios do estado da Bahia que possuem a Estratégia de Saúde da Família com equipe de saúde bucal e CEO implementados (Quadro 1). Na Bahia existem 80 CEO e 2.911 ESFSB, com cobertura SB AB de 66,30\% (Dados de nov. /2020, e-Gestor AB).

Os procedimentos odontológicos foram coletados do DATASUS- SIA/SUS (2020). Para análise do financiamento, foi utilizado o site da transparência do Fundo Nacional de Saúde, a base do SIOPS. Para oferta dos serviços os dados foram coletados do e-Gestor AB (coberturas SB) e do $1^{\circ}$ ciclo do PMAQ-CEO.

Quadro 1. Municípios eleitos para estudo segundo núcleo regional de saúde. Salvador, Bahia, 2021.

\begin{tabular}{|c|c|}
\hline $\begin{array}{l}\text { NÚCLEO REGIONAL } \\
\text { DE SAÚDE }\end{array}$ & MUNICÍPIOS \\
\hline Centro Leste & $\begin{array}{l}\text { Boa Vista do Tupim, Conceição do Coité, Feira de Santana, Iaçu, Ipirá, } \\
\text { Iraquara, Itaberaba, Itaeté, Pintadas, Ruy Barbosa, Santo Estevão, Utinga }\end{array}$ \\
\hline Centro Norte & Capim Grosso, Irecê, Jacobina, Mairi, Morro do Chapéu \\
\hline Extremo Sul & $\begin{array}{l}\text { Alcobaça, Eunápolis, Itabela, Itamaraju, Itanhém, Medeiros Neto, Nova } \\
\text { Viçosa, Porto Seguro, Prado, Teixeira de Freitas }\end{array}$ \\
\hline Leste & $\begin{array}{l}\text { Cachoeira, Camaçari, Candeias, Dias D’Ávila, Governador Mangueira, } \\
\text { Lauro de Freitas, Madre de Deus, Mata de São João, Pojuca, Salvador, } \\
\text { Santo Antônio de Jesus, São Félix, São Sebastião do Passé, Vera Cruz }\end{array}$ \\
\hline Nordeste & Alagoinhas, Catu \\
\hline Norte & Juazeiro, Paulo Afonso, Senhor do Bonfim \\
\hline Oeste & $\begin{array}{l}\text { Bom Jesus da Lapa, Canápolis, Correntina, Ibotirama, Luís Eduardo } \\
\text { Magalhães, Santa Maria da Vitória, Santa Rita de Cássia, São Desidério, } \\
\text { São Félix do Coribe }\end{array}$ \\
\hline Sudoeste & $\begin{array}{l}\text { Brumado, Caculé, Cândido Sales, Guanambi, Igaporã, Itapetinga, } \\
\text { Livramento de Nossa Senhora, Nova Canaã, Vitória da Conquista }\end{array}$ \\
\hline Sul & $\begin{array}{l}\text { Ilhéus, Ipiaú, Itabuna, Itajuípe, Jaguaquara, Jequié, Una, Wenceslau } \\
\text { Guimarães }\end{array}$ \\
\hline
\end{tabular}

Fonte: Elaboração própria.

Para análise do estudo foram considerados dois indicadores de utilização propostos para Saúde Bucal na Portaria $\mathrm{N}^{\circ}$ 1.631 de 1 de outubro de 2015, Ações básicas em Odontologia e Ações especializadas em Odontologia para a população geral. Estes indicadores permitem compreender como vem se configurando a relação entre $\mathrm{AB}$ e $\mathrm{AE}$ dos municípios e a oferta dos serviços odontológicos (Brasil, 2015). Esta portaria aprovou critérios e padrões para o planejamento e programação de ações e serviços de saúde no âmbito do SUS. A variável dependente refere-se ao cumprimento de $50 \%$ da meta para os dois padrões ( $\mathrm{AB}$ e $\mathrm{AE})$. 
Destaca-se que o cumprimento de meta está relacionado aos padrões propostos na portaria, para ações básicas valor mínimo de 1,5 procedimentos/hab/ano e para ações especializadas 0,05 procedimentos/hab/ano, quando o município cumpriu $50 \%$ dos dois padrões então cumpriu a meta de 50\%. Os resultados encontrados a partir destes padrões, são fundamentais para avaliação e monitoramento dos serviços de saúde bucal. A escolha dos 50\% se fundamenta na Resolução CIB No 503/2013, pois para o município implantar um CEO deve possuir 50\% ou mais de cobertura de saúde bucal na atenção básica.

Os dados de caracterização dos municípios foram extraídos do site do Instituto Brasileiro de Geografia e Estatística (IBGE, 2010): porte populacional, IDHM, índice de Gini, salário médio mensal dos trabalhadores formais, percentual da população com rendimento nominal mensal per capita de até $1 / 2$ salário mínimo, esgotamento sanitário adequado, urbanização de vias públicas.

O financiamento foi analisado segundo os valores nominais e deflacionados pelo Índice Nacional de Preços ao Consumidor Amplo (IPCA), a partir do Fundo Nacional de Saúde e dos indicadores disponíveis no SIOPS: participação da receita de impostos na receita total do Município; participação das transferências intergovernamentais na receita total do Município; participação \% das transferências para a Saúde (SUS) no total de recursos transferidos para o Município; despesa total com saúde - em R $\$ / h a b$., sob a responsabilidade do Município, por habitante; participação da despesa com investimentos na despesa total com saúde; participação das transferências para a saúde em relação à despesa total do município com saúde e participação da receita própria aplicada em Saúde conforme a Lei Complementar 141/2012.

Para oferta e organização dos serviços, a variável cobertura de saúde bucal foi extraída do e-Gestor e as variáveis, Tipo de CEO e quantidade de cirurgiões-dentistas que atuam nas especialidades mínimas com 67 respondentes, extraídas do módulo VII- Observação no CEO, $1^{\circ}$ ciclo PMAQ-CEO. As demais variáveis foram extraídas do módulo VIII - Gerente e cirurgião-dentista, $1^{\circ}$ ciclo PMAQ-CEO. O mecanismo de ingresso do cirurgião-dentista com 67 respondentes e as demais com 66 respondentes: existência de gerente no CEO, formação do gerente no CEO, monitoramento e análise das metas no CEO, CEO realiza matriciamento para as ESB da AB, CEO realiza ações clínicas junto as ESB da AB e forma de acesso ao CEO.

As variáveis independentes eleitas para a presente pesquisa foram agrupadas segundo: (i) as características demográficas do município; (ii) indicadores de financiamento, (iii) oferta dos serviços (Quadro 2). 
Quadro 2. Características demográficas, financiamento, cobertura, caracterização, gestão e acesso aos serviços odontológicos de municípios do Estado da Bahia, 2013-2019 (n=73). Salvador, Bahia, 2021.

\begin{tabular}{|c|c|c|}
\hline Dimensão & Variáveis & Categorias \\
\hline \multirow{7}{*}{$\begin{array}{l}\text { Caracterização } \\
\text { municipal }\end{array}$} & Porte populacional & Pequeno/ Médio/ Grande \\
\hline & IDHM & $\leq 0,637 />0,637$ \\
\hline & Índice de Gini & $\leq 0,55 />0,55$ \\
\hline & Salário Médio Mensal dos Trabalhadores Formais & $\leq 1,8 />1,8$ \\
\hline & $\begin{array}{l}\text { Rendimento nominal mensal per capita }=1 / 2 \\
\text { salário mínimo }\end{array}$ & $\leq 46,2 />46,2$ \\
\hline & Esgotamento sanitário adequado & $\leq 44,8 />44,8$ \\
\hline & Urbanização de vias públicas & $\leq 33 />33$ \\
\hline \multirow[t]{7}{*}{ Financiamento } & $\begin{array}{l}\text { Participação da receita de impostos na receita total } \\
\text { do município }\end{array}$ & $\leq 6,7 />6,7$ \\
\hline & $\begin{array}{l}\text { Participação das transferências } \\
\text { intergovernamentais na receita total do município }\end{array}$ & $\leq 85,9 />85,9$ \\
\hline & $\begin{array}{l}\text { Participação das Transferências para a Saúde } \\
\text { (SUS) no total de recursos transferidos para o } \\
\text { município }\end{array}$ & $\leq 14,4 />14,4$ \\
\hline & $\begin{array}{l}\text { Despesa total com Saúde por habitante, sob a } \\
\text { responsabilidade do município, em } \mathrm{R} \$ / \mathrm{hab} \text {. }\end{array}$ & $\leq 493,30 />493,30$ \\
\hline & $\begin{array}{l}\text { Participação da despesa com investimentos na } \\
\text { despesa total com Saúde }\end{array}$ & $\leq 3,8 />3,8$ \\
\hline & $\begin{array}{l}\text { Participação das transferências para a Saúde em } \\
\text { relação à despesa total do município com saúde }\end{array}$ & $\leq 54,1 />54,1$ \\
\hline & $\begin{array}{l}\text { Participação da receita própria aplicada em saúde } \\
\text { conforme a LC 141/2012 }\end{array}$ & $\leq 19,3 />19,3$ \\
\hline \multirow{4}{*}{$\begin{array}{l}\text { Caracterização, } \\
\text { cobertura e } \\
\text { utilização de } \\
\text { serviços }\end{array}$} & Cobertura de Saúde Bucal & $\leq 77,7 \% />77,7 \%$ \\
\hline & Tipo de CEO & I/ II III \\
\hline & $\begin{array}{l}\text { Quantidade de cirurgiões-dentistas nas } \\
\text { especialidades mínimas }\end{array}$ & $\leq 12 />12$ \\
\hline & $\begin{array}{l}\text { Mecanismo de ingresso de cirurgiões-dentistas } \\
\text { por concurso público }\end{array}$ & $\leq 3 />3$ \\
\hline \multirow{5}{*}{$\begin{array}{l}\text { Gestão do } \\
\text { serviço }\end{array}$} & Monitoramento e análise das metas no CEO & Sim/ Não \\
\hline & CEO realiza matriciamento para as ESB & Sim/ Não \\
\hline & $\begin{array}{l}\text { CEO realiza ações clínicas junto as ESB da } \\
\text { atenção básica }\end{array}$ & Sim/ Não \\
\hline & $\begin{array}{l}\text { Gerente no CEO e acumulação com atividade } \\
\text { profissional }\end{array}$ & $\begin{array}{l}\text { Sim, com acumulação/Sim, } \\
\text { sem acumulação/ Sem gerente }\end{array}$ \\
\hline & Formação do gerente do CEO & $\begin{array}{l}\text { Saúde coletiva/ gestão } \\
\text { pública/ outros/ sem formação }\end{array}$ \\
\hline Acesso & Forma de acesso ao CEO & $\begin{array}{l}\text { Espontânea/ referenciada/ } \\
\text { mista }\end{array}$ \\
\hline
\end{tabular}

Fonte: Elaboração própria.

Foi realizada uma análise exploratória, considerando a relação das variáveis independentes relacionadas às características demográficas dos municípios, aos indicadores de financiamento e oferta dos serviços de saúde bucal, com a variável resposta de cumprimento de $50 \%$ da meta. A homogeneidade entre as variáveis foi verificada utilizando-se o teste de qui quadrado de Pearson.

Por fim, foi procedida uma análise multivariada, do tipo backward, para obtenção de um modelo de associação das variáveis que mostraram diferenças estatisticamente significantes e o cumprimento de meta em questão. Foram calculadas as Odds Ratio (OR) tanto brutas quanto ajustadas pelas variáveis potencialmente intervenientes.

Para todas as análises foi considerado o valor de p-valor < 0,05, Intervalo de Confiança (IC) 95\%. Foi utilizado o pacote estatístico software STATA, versão 12. 
Essa pesquisa foi realizada unicamente com dados secundários, de domínio público, o que dispensou análise ética prévia do seu protocolo.

\section{Resultados}

Os resultados mostraram que, segundo as características demográficas dos 73 municípios estudados, 43 eram de pequeno porte, 16 de médio porte e 14 de grande porte, com maior cumprimento de meta naqueles de pequeno porte $(\mathrm{p}=0,02)$. No ano de 2010, o IDH da Bahia representou 0,660. Dos municípios em estudo, 38 (52,06\%) municípios representaram IDHM maior que 0,637 e $35(47,94 \%)$ representaram IDHM menor ou igual a 0,637. O índice de Gini foi maior que 0,55 em 39 $(53,43 \%)$ municípios com maior cumprimento de meta (Tabela 1$)$.

No trabalho e rendimento nos municípios, o salário médio mensal dos trabalhadores formais foi menor ou igual a 1,8 em 37 (50,68\%), obtendo maior cumprimento de meta. O rendimento nominal mensal per capita de até 1/2 salário-mínimo se apresentou maior que 46,2\% para 39 (53,43\%) municípios com maior cumprimento de meta (Tabela 1$)$.

Tabela 1. Características demográficas dos municípios do Estado da Bahia, 2013-2019 (n=73). Salvador, Bahia, 2021.

\begin{tabular}{|c|c|c|c|c|c|}
\hline \multirow{3}{*}{ Características Demográficas } & \multicolumn{5}{|c|}{ Cumprimento maior que $50 \%$ da meta } \\
\hline & \multicolumn{2}{|c|}{ Sim } & \multicolumn{2}{|c|}{ Não } & \multirow[t]{2}{*}{ p-valor } \\
\hline & $\mathrm{n}$ & $\%$ & $\mathrm{n}$ & $\%$ & \\
\hline \multicolumn{6}{|l|}{ Porte populacional } \\
\hline Pequeno & 27 & 62,79 & 16 & 37,21 & 0,02 \\
\hline Médio & 5 & 35,25 & 11 & 68,75 & \\
\hline Grande & 4 & 28,57 & 10 & 71,43 & \\
\hline \multicolumn{6}{|l|}{ IDHM } \\
\hline$\leq 0,637$ & 18 & 51,43 & 17 & 48,57 & 0,73 \\
\hline$>0,637$ & 18 & 47,37 & 20 & 52,63 & \\
\hline \multicolumn{6}{|l|}{ Índice de Gini } \\
\hline$\leq 0,55$ & 16 & 47,06 & 18 & 52,94 & 0,72 \\
\hline$>0,55$ & 20 & 51,28 & 19 & 48,72 & \\
\hline \multicolumn{6}{|l|}{ Salário Médio Mensal dos Trabalhadores } \\
\hline \multicolumn{6}{|l|}{ Formais } \\
\hline$\leq 1,8$ & 19 & 51,35 & 18 & 48,65 & 0,72 \\
\hline$>1,8$ & 17 & 47,22 & 19 & 52,78 & \\
\hline \multicolumn{6}{|c|}{$\begin{array}{l}\text { Rendimento nominal mensal per capita } \\
\leq \frac{1}{2} \text { salário mínimo }\end{array}$} \\
\hline$\leq 46,2$ & 17 & 50,00 & 17 & 50,00 & 0,91 \\
\hline$>46,2$ & 19 & 48,72 & 20 & 51,28 & \\
\hline \multicolumn{6}{|l|}{ Esgotamento sanitário adequado } \\
\hline$\leq 44,8$ & 21 & 56,76 & 16 & 43,24 & 0,20 \\
\hline$>44,8$ & 15 & 41,67 & 21 & 58,33 & \\
\hline \multicolumn{6}{|l|}{ Urbanização de vias públicas } \\
\hline$\leq 33$ & 32 & 47,76 & 35 & 52,24 & 0,37 \\
\hline$>33$ & 14 & 66,67 & 12 & 33,33 & \\
\hline
\end{tabular}

*Ano de referência dos dados analisados: 2010 (IBGE).

Fonte: Elaboração própria baseado no site do IBGE (www.ibge.com.br).

Em 2010, o esgotamento sanitário adequado representou um número menor ou igual a 44,8\% para 37 municípios (50,68\%) e maior cumprimento de meta. Na urbanização de vias públicas, 67 municípios $(91,78 \%)$ apresentaram taxa menor ou igual a $33 \%$ obtendo melhor cumprimento da meta (Tabela 1).

Quanto ao financiamento, a participação da receita de impostos na receita total dos municípios observou-se que foi menor ou igual a 6,7\% em 37 (50,68\%) municípios. As transferências intergovernamentais na receita total representaram uma 
taxa maior que 85,9\% em 37 municípios $(50,68 \%)$ e estes cumpriram a meta, sendo a variável estatisticamente significante $(\mathrm{p}=0,03)($ Tabela 1$)$.

A participação das transferências para a saúde (SUS) no total de recursos transferidos foi em média > 14,4\% nos 37 (50,69\%) municípios versus $\leq 14,4 \%$ nos 36 (49,31\%) restantes (Tabela 2).

Tabela 2. Percentual de Cumprimento das Metas versus financiamento da atenção à saúde bucal no Estado da Bahia, 20132019 (n=73). Salvador, Bahia, 2021.

\begin{tabular}{|c|c|c|c|c|}
\hline \multirow{3}{*}{ Financiamento } & \multicolumn{4}{|c|}{ Cumprimento da meta $>\mathbf{5 0 \%}$} \\
\hline & Sim & & & $\begin{array}{c}\text { p- } \\
\text { valor }\end{array}$ \\
\hline & $\%$ & $\mathrm{n}$ & $\%$ & \\
\hline
\end{tabular}

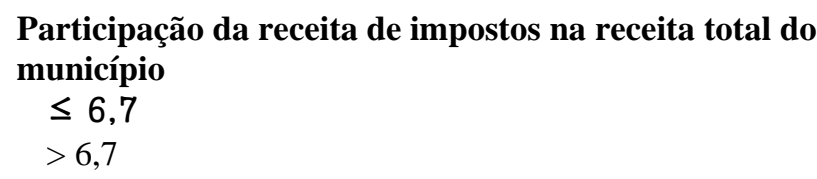

Participação das transferências intergovernamentais na receita total do município

$\begin{array}{llllll}\leq 85,9 & 13 & 36,11 & 23 & 63,89 & 0,03 \\ >85,9 & 23 & 62,16 & 14 & 37,84 & \end{array}$

Participação das Transferências para a Saúde (SUS) no total de recursos transferidos para o município $\leq 14,4$

$21 \quad 56,76 \quad 16 \quad 43,24 \quad 0,20$

$15 \quad 41,67 \quad 21 \quad 58,33$

$>14,4$

$\begin{array}{llll}21 & 58,33 & 15 & 41,67 \\ 15 & 40,54 & 22 & 59,46\end{array}$

0,13

Despesa total com Saúde por habitante, sob a responsabilidade do município, em $\mathbf{R} \$ / \mathbf{h a b}$.

$\begin{array}{llllll}\leq 493,30 & 17 & 45,95 & 20 & 54,05 & 0,56\end{array}$

$>493,30$

$19 \quad 52,78 \quad 17 \quad 47,22$

Participação da despesa com investimentos na despesa total com Saúde

$\begin{array}{llllll}\leq 3,8 & 18 & 51,43 & 17 & 48,57 & 0,73 \\ >3,8 & 18 & 47,37 & 20 & 52,63 & \end{array}$

\section{Participação das transferências para a Saúde em relação à} despesa total do município com saúde $\leq 54,1$

$\begin{array}{llll}20 & 55,56 & 16 & 44,44 \\ 16 & 43,24 & 21 & 56,76\end{array}$

0,30

$>54,1$

$16 \quad 43,24 \quad 21 \quad 56,76$

Participação da receita própria aplicada em saúde conforme a LC141/2012 (mínimo de $15 \%$ para municípios) $\leq 19,3$

$\begin{array}{lllll}17 & 47,22 & 19 & 52,78 & 0,72 \\ 19 & 51,35 & 18 & 48,65\end{array}$

$>19,3$

Fonte: Elaboração própria baseado no site do SIOPS (http://siops.datasus.gov.br/relindicadoresmun2.php?escmun=3).

Já no tocante à despesa total em saúde por habitante, 37 municípios gastaram até $\mathrm{R} \$ 493,30$ reais por habitante. Destaca-se que na participação da despesa com investimentos na despesa total com saúde, 35 municípios apresentaram- se menor ou igual à média de 3,8\% e 38 municípios maior que 3,8\%, tendo a mesma representatividade no cumprimento de meta. Na receita própria aplicada em saúde, 37 (50,68\%) municípios participaram com mais de 19,3\% e cumpriram melhor a meta (Tabela 2). 
Com relação à oferta de serviços odontológicos, dos 73 municípios estudados, 37 (50,68\%) municípios representaram cobertura de saúde bucal menor ou igual a 77,7\% e $36(49,32 \%)$ maior que 77,7\% tendo este grupo maior cumprimento de meta e a variável significância estatística $\mathrm{p}=0,001$. No que se refere ao tipo de CEO, 32 municípios tinham CEO tipo I, 36 com CEO tipo II e apenas 5 possuíam CEO tipo III, com melhor cumprimento de meta nos de tipo I ( $p=0,01)$ (Tabela 3 ).

Por outro lado, dentre os 66 municípios analisados, quanto à existência de gerente no CEO, percebeu-se que apenas 12 municípios não possuíam gerente, e naqueles em que o profissional acumulava a atividade clínica e de gerente existia maior cumprimento de meta. Destes gerentes, 53 não possuíam formação nem em saúde coletiva, nem em gestão pública, 11 possuíam em saúde coletiva e 2 em gestão pública, tendo maior resposta quando o gerente apresentou outro tipo de formação (Tabela 3).

Já a quantidade de cirurgiões-dentistas nas especialidades mínimas, cirurgia oral, endodontia, estomatologia, pacientes com necessidades especiais e periodontia representou uma média de 12 dentistas, sendo que a maioria $(86,30 \%)$ correspondeu com menor ou igual a 12 dentistas e cumpriram melhor a meta. O mecanismo de ingresso do cirurgião-dentista por concurso público mostrou uma média de 3 cirurgiões-dentistas. Destes, 45 municípios (67,16\%) possuíam média menor ou igual a 3 e cumpriram melhor a meta e $22(32,84 \%)$ maior que 3 (Tabela 3$)$. 
Tabela 3. Oferta de serviços odontológicos no Estado da Bahia, 2013-2019 (n=73). Salvador, Bahia, 2021.

\begin{tabular}{|c|c|c|c|c|c|}
\hline \multirow{3}{*}{ Características } & \multicolumn{5}{|c|}{ Cumprimento maior que $50 \%$ da meta } \\
\hline & \multicolumn{2}{|c|}{ Sim } & \multicolumn{2}{|c|}{ Não } & \multirow{2}{*}{$\begin{array}{c}\text { p- } \\
\text { valor }\end{array}$} \\
\hline & $\mathrm{n}$ & $\%$ & $\mathrm{n}$ & $\%$ & \\
\hline \multicolumn{6}{|l|}{ Cobertura de Saúde Bucal $(n=73)$} \\
\hline$\leq 77,7 \%$ & 11 & 29,73 & 26 & 70,27 & \multirow[t]{2}{*}{0,001} \\
\hline$>77,7 \%$ & 25 & 69,44 & 11 & 30,56 & \\
\hline \multicolumn{6}{|l|}{ Tipo de CEO $(n=73)$} \\
\hline I & 21 & 65,63 & 11 & 34,38 & \multirow[t]{3}{*}{0,01} \\
\hline II & 15 & 41,67 & 21 & 58,33 & \\
\hline III & 0 & 00,00 & 5 & 100,00 & \\
\hline \multicolumn{6}{|l|}{$\begin{array}{l}\text { Gerente no CEO e acumulação de } \\
\text { atividade profissional }(n=66)\end{array}$} \\
\hline Sim/ com acumulação & 16 & 64,00 & 9 & 36,00 & \multirow[t]{3}{*}{0,25} \\
\hline Sim/ sem acumulação & 12 & 41,38 & 17 & 58,62 & \\
\hline Sem gerente & 6 & 50,00 & 6 & 50,00 & \\
\hline \multicolumn{6}{|l|}{ Formação do gerente do CEO $(n=66)$} \\
\hline Sim, em saúde coletiva & 4 & 36,36 & 7 & 63,64 & \multirow[t]{4}{*}{0,72} \\
\hline Sim, em gestão pública & 1 & 50,00 & 1 & 50,00 & \\
\hline Sim, outros & 17 & 56,67 & 13 & 43,33 & \\
\hline Não & 12 & 52,17 & 11 & 47,83 & \\
\hline \multicolumn{6}{|c|}{$\begin{array}{l}\text { Quantidade de cirurgiões- dentistas nas } \\
\text { especialidades mínimas (n=67) }\end{array}$} \\
\hline$\leq 12$ & 32 & 50,79 & 31 & 49,21 & \multirow[t]{2}{*}{0,97} \\
\hline$>12$ & 2 & 50,00 & 2 & 50,00 & \\
\hline \multicolumn{6}{|l|}{$\begin{array}{l}\text { Ingresso de cirurgiões- dentistas por } \\
\text { concurso público }(n=67)\end{array}$} \\
\hline$\leq 3$ & 25 & 55,56 & 20 & 44,44 & \multirow[t]{2}{*}{0,26} \\
\hline$>3$ & 9 & 40,91 & 13 & 59,09 & \\
\hline \multicolumn{6}{|l|}{ Monitoramento e análise das metas no } \\
\hline $\operatorname{CEO}(n=66)$ & & & & & \multirow{3}{*}{0,18} \\
\hline Sim & 24 & 47,06 & 27 & 52,94 & \\
\hline Não & 10 & 66,67 & 5 & 33,33 & \\
\hline \multicolumn{6}{|c|}{$\begin{array}{l}\text { CEO realiza matriciamento para as ESB } \\
\text { da atenção básica }(n=66)\end{array}$} \\
\hline Sim & 25 & 52,08 & 23 & 47,92 & \multirow[t]{2}{*}{0,88} \\
\hline Não & 9 & 50,00 & 9 & 50,00 & \\
\hline \multicolumn{6}{|c|}{$\begin{array}{l}\text { CEO realiza ações clínicas junto as ESB } \\
\text { da atenção básica }(n=66)\end{array}$} \\
\hline Sim & 18 & 48,65 & 19 & 51,35 & \multirow[t]{2}{*}{0,60} \\
\hline Não & 16 & 55,17 & 13 & 44,83 & \\
\hline \multicolumn{6}{|l|}{ Forma de acesso ao CEO $(n=66)$} \\
\hline Espontânea & - & - & - & - & \multirow{3}{*}{0,65} \\
\hline Mista (espontânea e referenciada) & 21 & 53,85 & 18 & 46,15 & \\
\hline Referenciada & 13 & 48,15 & 14 & & \\
\hline
\end{tabular}

Além disso, verificou-se que o monitoramento e análise das metas no CEO foi realizado por 51 (77,27\%) municípios, prevalecendo o maior cumprimento de meta, enquanto que somente 15 não o fizeram. Na relação $\mathrm{CEO}$ e $\mathrm{AB}$, referente ao matriciamento, 48 (72,72\%) municípios realizaram, e em 37 (56,06\%) municípios realizaram ações clínicas conjuntas. Quando ocorria o matriciamento e as ações conjuntas a meta se apresentava mais expressiva. A maior parte do acesso ao CEO se dava 
por demanda mista (espontânea e referenciada) em 39 (59,10\%) municípios com maior cumprimento de meta, e de forma referenciada nos demais 27 (40,90\%) municípios (Tabela 3).

A partir da análise multivariada, foi observada associação estatisticamente significante entre cobertura de saúde bucal e cumprimento de meta menor ou igual a 50\% nos modelos brutos e quando ajustados por porte populacional, IDHM, índice Gini, despesa total com saúde por hab., quantidade de especialistas, ingresso no serviço por concurso público, despesa total com saúde por habitante, salário médio mensal dos trabalhadores formais e urbanização de vias públicas (OR bruta $=5,37$; IC95\% $[1,98-14,60] /$ OR ajustada $=6,27$; IC95\% [1,52 - 25,81] (Tabela 4).

Tabela 4. Odds Ratio (OR) e Intervalo de confiança (IC 95\%) obtidos mediante regressão logística não condicional para a associação entre cobertura de saúde bucal e cumprimento de até 50\% da meta no Estado da Bahia, Brasil, 2021 (n=73).

\begin{tabular}{ccccc}
\hline Cobertura de Saúde Bucal & MODELOS & OR & IC 95\% & p* $^{*}$ \\
\hline $\begin{array}{c}\text { Cumprimento } \\
\text { Meta }\end{array}$ & Bruto (n=73) & 5,37 & $(1,98-14,60)$ & 0,001 \\
& Ajustado ${ }^{1}(\mathrm{n}=73)$ & 6,27 & $(1,52-25,81)$ & 0,011 \\
\hline
\end{tabular}

* Valor de significância estatística: $\mathrm{p} \leq 0,05$.

Fonte: Dados do IBGE (2010), SIOPS (2020), $1^{\circ}$ ciclo do PMAQ-CEO e site do e-Gestor AB (2020).

\section{Discussão}

O presente estudo revelou que uma menor cobertura de saúde bucal está positivamente associada ao não cumprimento de 50\% da meta de utilização dos serviços odontológicos na $\mathrm{AB}$ e $\mathrm{AE}$ dos municípios com CEO do estado da Bahia no período 2013-2019. Além disso, tal associação se manteve, mesmo depois de realizados os ajustes por porte populacional, IDHM, índice Gini, despesa total com saúde por hab., quantidade de especialistas, ingresso no serviço por concurso público, despesa total com saúde por habitante, salário médio mensal dos trabalhadores formais e urbanização de vias públicas.

Sabe-se que muitos fatores influenciam a utilização de serviços, a exemplo das características demográficas dos municípios. Naqueles de pequeno e médio porte houve maior cumprimento da meta do que os de grande porte, e quanto ao IDHM, locais com índice menor ou igual e maior que 0,637 representaram o mesmo cumprimento de meta. No entanto, resultado diferente foi observado por Figueiredo e Goes (2009), por exemplo, vez que verificaram que quanto menor o porte populacional e o desenvolvimento humano, pior era o desempenho do CEO. Observa-se que esta divergência pode estar relacionada ao baixo número de municípios no presente estudo ou pode indicar que municípios menores tenham mais facilidade na integralidade das ações já que possuem um número menor de serviços a serem administrados, garantindo melhor os fluxos.

Considerando municípios de grande porte, com IDHM maior que 0,7 e com cobertura de saúde bucal de até quatro mil habitantes, Neves et al. (2019) observaram maior realização de procedimentos odontológicos curativos. No estudo de Galvão e Roncalli (2021), ao avaliar os fatores associados ao desempenho dos municípios quanto à disponibilidade de serviços odontológicos especializados, foi identificado que aqueles com menor porte populacional apresentaram maior proporção de municípios com maior desempenho. Com relação ao IDHM, os autores verificaram que aqueles com menor IDHM apresentaram maior proporção de municípios com maior desempenho. Por sua vez, os municípios com menor renda per capita apresentaram em sua maior parte melhor desempenho (Galvão \& Roncalli, 2021). Estes achados mostram que os indicadores sociais e as características demográficas podem influenciar na utilização e desempenho dos serviços. 
Segundo Neves et al. (2019), as desigualdades socioeconômicas impactam diretamente a saúde. Em relação às desigualdades sociais e a condição de saúde bucal, estas, geram iniquidades que são minimizadas devido às políticas de saúde existentes (Fernandes, Pinho, Queiroz, \& Thomaz, 2016). Sabe-se que mesmo com a inserção da ESB no PSF, as condições de vida dos indivíduos refletem na utilização dos serviços de saúde bucal configurando um agente modulador da saúde bucal nos diferentes municípios.

Um exemplo disso foi o aumento das ESB e a expansão dos CEO verificados, a despeito da persistência de várias dificuldades com relação à utilização de serviços nos municípios e o financiamento dessas ações. Rossi, Sobrinho, Chaves, \& Martelli (2019), relataram que os investimentos financeiros no SUS, a nível federal, apresentaram crescimento entre 2003 a 2010 e estabilidade entre 2011 a 2018. Na atenção básica os repasses foram crescentes até 2012, logo após sofreram redução, manutenção e queda em 2018, enquanto na atenção especializada tais repasses foram crescentes até 2017. O fato é que, desde que o SUS existe, há um subfinanciamento, e uma não constância nos repasses, e o que se tem nesse momento é, na verdade, uma limitação dos gastos públicos (Rossi et al., 2018; Menezes, Moretti, \& Reis, 2019).

Por sua vez, os gastos públicos segundo o indicador despesa total em saúde por habitante no Brasil apresentou crescimento ao longo dos anos. No ano de 1999 era de $\mathrm{R} \$ 83,70$, em $2010 \mathrm{R} \$ 388,40$ chegando em 2019 com $\mathrm{R} \$ 441,88$ (Celeste, Moura, Santos, \& Tovo, 2014; Corrêa \& Celeste, 2015; CFM, 2020). O estado da Bahia, em 2019, representou uma das últimas posições, ficando à frente apenas do Maranhão e Pará, com uma despesa de R \$291,97 por habitante. Já a realidade da capital Salvador, tem um dos menores desempenhos com gasto de $\mathrm{R} \$ 275,56$, tendo Teresina, capital do Piauí, a primeira posição com gasto de R \$703,16 (CFM, 2019). Deve-se considerar a importância de um maior gasto com saúde por habitante nos municípios, já que segundo o estudo, desse modo há um maior cumprimento de meta.

Logo, estes dados podem justificar porque no presente estudo a maioria dos municípios mostraram uma média igual ou menor que $\mathrm{R} \$ 493,30$. Nota-se que mesmo com essa média, os achados referentes à Bahia e sua capital Salvador apontam para um baixo investimento em saúde por habitante. Em comparação com o estudo de Galvão e Roncalli (2021) sobre os municípios brasileiros com CEO, a média de gastos por habitante foi de $\mathrm{R} \$ 599,40$, em que $50 \%$ dos municípios avaliados gastaram até $\mathrm{R} \$ 531,70$ por habitante. Estes valores na despesa total com saúde por habitante podem refletir as dificuldades encontradas pelos serviços para o cumprimento das metas. Destaca-se que o Brasil apesar de possuir um sistema público de saúde de acesso universal, ainda apresenta gastos maiores com o setor privado em saúde como porcentagem do PIB (4,5\%), comparado ao gasto no setor público em saúde como porcentagem do PIB (3,8\%) (Figueiredo, Prado, Medina, \& Paim, 2018).

A participação das transferências intergovernamentais na receita total do município pode refletir o grau de dependência do município em relação às transferências de outras esferas do governo, ressaltando assim que a indução federal é fundamental para o cumprimento das metas estabelecidas. No dado estudo, municípios com participação maior que $85,9 \%$ cumpriram melhor a meta, porém isto pode indicar que estes têm maior dependência com respeito às transferências de recursos de outras esferas de governo (SIOPS, 2009). Ressalta-se a importância das transferências intergovernamentais para a saúde, pois pode promover uma redistribuição de recursos ao longo do território e atribuir maior capacidade e responsabilidade de gestão aos governos locais (Simão \& Orellano, 2015). Dessa forma, essa dependência pode influenciar diretamente no cumprimento de meta pelos municípios.

Outro quesito relevante considerado na presente investigação, foi a identificação dos municípios que implementaram CEO na Bahia e suas características. Ao analisar a cobertura de saúde bucal, Barros et al. (2016), em estudo com dois municípios do Estado da Bahia, observou que no município A, os residentes de área com cobertura de SB utilizaram mais os serviços ofertados se comparado ao município B, em que as áreas descobertas utilizaram mais. No presente estudo, foi observado que os municípios com cobertura de SB maior que 77,7\% cumpriram melhor a meta estabelecida se comparado aos com menor ou igual a $77,7 \%$. 
A análise multivariada mostrou que uma menor cobertura de saúde bucal reflete significativamente ao não cumprimento de 50\% da meta. Esses achados sugerem que quanto maior for a cobertura, maior será o acesso a serviços de SB da população. Este efeito associado ao aumento de cobertura de SB permaneceu mesmo após controle por fatores demográficos, de financiamento e oferta de serviços odontológicos. Estes achados corroboram com os de Côrreia e Celeste (2015), sugerindo que o aumento da cobertura de SB parece ser uma estratégia efetiva para o aumento de indicadores de utilização de serviços odontológicos.

Assim, reafirma-se que o aumento de cobertura de saúde bucal na AB é de suma importância, pois se associa a um maior cumprimento de meta e consequente maior utilização dos serviços especializados, por meio do fluxo de referência e contrarreferência. Por outro lado, a forma de acesso ao CEO representou maiores valores no cumprimento de meta quando a demanda foi mista (espontânea e referenciada), destacando assim, a importância da garantia do fluxo dos serviços de $\mathrm{AB}$ e $\mathrm{AE}$. A baixa cobertura de SB pode sinalizar problemas como tempo de espera, presença de barreiras geográficas, escassez de recursos humanos e dificuldades na comunicação entre os profissionais da atenção básica e especializada (Celeste et al., 2014). Fundamenta-se que para efetivação da $\mathrm{AB}$ é essencial a promoção da saúde, pois objetiva a melhoria na qualidade de vida e a redução da vulnerabilidade e riscos à saúde, levando a população a ter melhorias no modo de viver: condições de trabalho, habitação, educação, lazer, cultura, acesso a bens e serviços essenciais (Brasil, 2008). Dessa maneira, garantir a cobertura na $\mathrm{AB}$ é primordial para um aumento da utilização dos serviços especializados e integralidade do cuidado.

Quanto ao tipo de CEO, a maioria foi do tipo I ou II, apontando para um maior cumprimento de meta nos de tipo I, sendo os do tipo III com $n=5$ e sem cumprimento de meta. Porém, outro estudo sugere que os serviços mais estruturados garantiram um maior cumprimento da atenção especializada (Goes et al., 2012). Ressalta-se que esta divergência com relação a estrutura do serviço não é o principal entrave para avaliação e cumprimento de meta.

E por fim, destaca-se que a organização dos serviços de saúde depende de uma rede regionalizada, necessitando fluxos entre os diferentes níveis de atenção, para garantir a integralidade do atendimento e das ações de saúde (Paiva, Randow, Diniz, \& Guerra, 2018). Neste sentido, o papel do gestor é fundamental, pois este age como articulador e organizador dos serviços sendo um importante instrumento para efetivação das políticas (Silva \& Roquete, 2013). O presente estudo aponta a importância do gerente no CEO, muitas vezes atuando exclusivamente como gerente. Dos 66 respondentes, 54 municípios possuíam gestores, e observou-se que quando existia o profissional, houve maior cumprimento de meta.

Outro fator relevante se refere à formação destes profissionais, apenas 11 apresentaram formação em saúde coletiva e 2 em gestão pública. Os demais 53 tinham outro tipo de formação ou nenhuma. Estes achados corroboram com estudo de Lorenzetti, Lanzoni, Assuiti, Pires, e Ramos (2014), que mostra que mais de 70\% dos profissionais que ocupam posição de gestores não têm formação ou fizeram alguma capacitação para tal cargo. Além disso, a competência do gestor está diretamente relacionada com a formação, pois esta influência no modo de operacionalização das estratégias e na dinâmica das equipes dos serviços de saúde (Silva \& Roquete, 2013). Desta maneira, estes achados refletem a necessidade de estratégias para fortalecer o embasamento teórico dos gestores e sua aproximação com os instrumentos de gestão em saúde (Ferreira et al., 2018).

\section{Considerações Finais}

As desigualdades na oferta e utilização de serviços odontológicos persistem como um desafio a ser superado. Isso tem sido relacionado a diversos fatores tais como os ora detectados, a saber, características demográficas, de financiamento, de cobertura, gestão e acesso a tais serviços. Percebeu-se, por exemplo, que os gastos com saúde por habitante ainda são pouco expressivos na Bahia, o que pode interferir no cumprimento de metas. Além disso, notou-se que municípios que tiveram maior 
participação das transferências intergovernamentais na receita total, obtiveram melhores resultados quanto ao cumprimento de meta.

$\mathrm{Na}$ organização dos serviços, reafirma-se a importância do aumento de cobertura de saúde bucal na $\mathrm{AB}$, pois se associa a uma maior utilização dos serviços especializados, garantindo a integralidade do cuidado. Isso porque os presentes achados reafirmam o impacto da baixa cobertura de saúde bucal contribuindo no não cumprimento de $50 \%$ da meta nos municípios.

Da mesma forma, a formação do gestor se faz necessária para garantir um melhor desempenho na gestão do serviço. Destaca-se a importância da educação permanente nas distintas estratégias presenciais e de forma remota no âmbito estadual e municipal.

Embora achados muito valiosos tenham sido revelados no presente estudo, consideram-se como limitações do mesmo a utilização de dados secundários não sendo possível garantir a qualidade das informações, bem como os padrões propostos pela portaria $\mathrm{N}^{\mathrm{o}} 1.631$ de 1 de outubro de 2015, são distantes da realidade e singularidade de cada município, dificultando o cumprimento das metas. Ressalta-se também, como limitação, o tamanho da amostra do estudo $n=73$ municípios, e a não inclusão do ano de 2020 devido à pandemia do COVID-19. Aponta-se que neste estudo adotou-se o desfecho baseado na referência mínima para implantação de um CEO definido pelo estado da Bahia, ou seja, um ato formal do Estado.

Entretanto, a abordagem utilizada é de extrema importância para compreensão dos fatores municipais que interferem na utilização dos serviços e consequente cumprimento de metas. Outros desfechos podem ser assumidos em estudos futuros baseados em pesquisas avaliativas ou outros quadros teóricos de referência.

\section{Referências}

Aragão, N. S. C., Amorim, M. M., \& Conceição S. S. (2019). Financiamento da Política Nacional de Saúde Bucal: um estudo comparativo entre dois municípios da Bahia. Revista Brasileira De Saúde Funcional, 9(1), 17.

Baldani, M. H., Ribeiro, A. E., Gonçalves, J. R. S. N., \& Ditterich, R.G. (2018). Processo de trabalho em saúde bucal na atenção básica: desigualdades intermunicipais evidenciadas pelo PMAQ-AB. Saúde em Debate, 42(1), 145-162.

Brasil. (1990). Lei $n^{\circ} 8.080$, de 19 de setembro de 1990. Lei Orgânica da Saúde. Dispõe sobre as condições para a promoção, proteção e recuperação da saúde, a organização e o funcionamento dos serviços correspondentes e dá outras providências. Brasília, set.

Brasil. (2004). Ministério da Saúde. Secretaria de Atenção à Saúde. Departamento de Atenção Básica. Coordenação de Saúde Bucal. Diretrizes da Política Nacional de Saúde Bucal. Brasília.

Brasil. (2008). Ministério da Saúde. Caderno de Atenção Básica, $n^{\circ}$ 17. Saúde Bucal. Brasília-DF.

Brasil. Constituição (2012). Lei $n^{\circ} 141$, de 13 de janeiro de 2012. Regulamenta O $§ 30$ do Art. 198 da Constituição Federal Para Dispor Sobre Os Valores Mínimos A Serem Aplicados Anualmente Pela União, Estados, Distrito Federal e Municípios em Ações e Serviços Públicos de Saúde; Estabelece Os Critérios de Rateio dos Recursos de Transferências Para A Saúde e As Normas de Fiscalização, Avaliação e Controle das Despesas Com Saúde nas 3 (Três) Esferas de Governo; Revoga Dispositivos das Leis nos 8.080, de 19 de Setembro de 1990, e 8.689, de 27 de Julho de 1993; e Dá Outras Providências. Brasília.

Brasil. (2013). Comissão Intergestores Bipartite da Bahia. Aprova critérios para habilitação de Centros de Especialidades Odontológicas - CEO e define fluxo de credenciamento destes serviços no Estado da Bahia. Resolução CIB N ${ }^{o}$ 503/2013, 12 de novembro de 2013.

Brasil. (2015). Perfil Socioeconômico da Bahia. Banco do Nordeste do Brasil.

Brasil. (2015). Ministério da Saúde. Aprova critérios e parâmetros para o planejamento e programação de ações e serviços de saúde no âmbito do SUS. Portaria n.1.631, de outubro de 2015.

Barros, S. G., Cangussu, M. C. T., Cruz, D. N., Silva, L. O. R., \& Roncalli, A. G. (2016). Impacto da implantação das equipes de saúde bucal na estratégia saúde da família em dois municípios do estado da Bahia. Revista de Saúde Coletiva da UEFS, 6(2), 37-42.

Celeste, R. K., Moura, F. R. R., Santos, C. P., \& Tovo, M. F. (2014). Análise da produção ambulatorial em municípios com e sem centros de especialidades odontológicas no Brasil em 2010. Cadernos de Saúde Pública, 30(3), 511-521.

CFM. Conselho Federal de Medicina (2020). Brasil gasta $R \$ 3,83$ ao dia com a saúde de cada habitante. https://portal.cfm.org.br/noticias/brasil-gasta-r-383ao-dia-com-a-saude-de-cada-habitante-2/

CFM. Conselho Federal de Medicina (2019). Salvador gasta R\$ 245 por habitante na saúde, diz CFM. Metro1. https://www.metro1.com.br/noticias/saude/67524,salvador-gasta-r-245-por-habitante-na-saude-diz-cfm. 
Chaves, S. C. L., Cruz, D. N., Barros, S. G., \& Figueiredo, A. L. (2011). Avaliação da oferta e utilização de especialidades odontológicas em serviços públicos de atenção secundária na Bahia, Brasil. Cadernos de Saúde Pública, 27(1), 143-154.

Corrêa, G. T., \& Celeste, R. K. (2015). Associação entre a cobertura de equipes de saúde bucal na saúde da família e o aumento na produção ambulatorial dos municípios brasileiros, 1999 e 2011. Cadernos de Saúde Pública, 31(12), 2588-2598.

Fernandes, J. K. B., Pinho, J. R. O., Queiroz, R. C. S., \& Thomaz, E. B. A. F. T. (2016). Avaliação dos indicadores de saúde bucal no Brasil: tendência evolutiva pró-equidade? Cadernos de Saúde Pública, 32(2).

Ferreira, J., CeluppI, I. C., Baseggio, L., Geremia, D. S., Madureira, V. S. F., \& Souza, J. B. (2018). Planejamento regional dos serviços de saúde: o que dizem os gestores? Saúde e Sociedade, 27(1), 69-79.

Figueiredo, N., \& Goes, P. S. A. (2009). Construção da atenção secundária em saúde bucal: um estudo sobre os Centros de Especialidades Odontológicas em Pernambuco, Brasil. Cad. Saúde Pública, Rio de Janeiro, 25(2), 259-67.

Figueiredo, J. O., Prado, N. M. B. L., Medina, M. G., \& Paim, J. S. (2018). Gastos público e privado com saúde no Brasil e países selecionados. Saúde em Debate, 42(2), 37-47.

Goes, P. S. A., Figueiredo, N., Neves, J. C., Silveira, F. M. M., Costa, J. F. R., Junior, G. A. P., \& Rosales, M. S. (2012). Avaliação da atenção secundária em saúde bucal: uma investigação nos centros de especialidades do Brasil. Cadernos de Saúde Pública, 28, 81-89.

Galvão, M. H. R., \& Roncalli, A. G. (2021). Desempenho dos municípios brasileiros quanto à oferta de serviços especializados em saúde bucal. Cadernos de Saúde Pública, 37(1), 30-40.

Gonçalves, J. R. S. N., Ditterich, R. G., Gonçalves, R. N., Gavina, V. P., Silva, R. O. C., Graziani, G. F., \& Kusma, S. Z. (2018). Desempenho dos Centros de Especialidades Odontológicas da $2^{\mathrm{a}}$ Regional de Saúde do Paraná. Cadernos Saúde Coletiva, 26(4), 432-438.

IBGE. Cidades e Estados. (2010). Disponível em: https://www.ibge.gov.br/cidades-e-estados/ba.html. Acesso em: 15 nov. 2020.

Kornis, G. E. M., Maia, L. S., \& Fortuna, R. F. P. (2011). Evolução do financiamento da atenção à saúde bucal no SUS: uma análise do processo de reorganização assistencial frente aos incentivos federais. Physis: Revista de Saúde Coletiva, 21(1), 197-215.

Lorenzetti, J., Lanzoni, G. M. M., Assuiti, L. F. C., Pires, D. E. P., \& Ramos, F. R. S. (2014). Gestão em saúde no Brasil: diálogo com gestores públicos e privados. Texto Contexto Enferm, v.23, n.2, p. 417-25.

Machado, F. C. A., Silva, J. V., \& Ferreira, M. A. F. (2015). Fatores relacionados ao desempenho de Centros de Especialidades Odontológicas. Ciência \& Saúde Coletiva, 20(4), 1149- 1163.

Mattos, G. C. M., Ferreira, E. F., Leite, I. C. G., \& Greco, R. M. (2014). A inclusão da equipe de saúde bucal na Estratégia Saúde da Família: entraves, avanços e desafios. Ciência \& Saúde Coletiva, 19(2), 373-382.

Menezes, A. P. R., Moretti, B., \& Reis, A. A. C. (2019). O futuro do SUS: impactos das reformas neoliberais na saúde pública - austeridade versus universalidade. Saúde em Debate, 43(5), 58-70.

Ministério da Saúde. (2019). Secretaria de Atenção Primária à Saúde. Informação e gestão da atenção básica, e-Gestor AB. https://egestorab.saude.gov.br/paginas/acessoPublico/relatorios/relHistoricoCoberturaSB.xhtml.

Neves, M., Giordani, J. M. A., \& Hugo, F. N. (2019). Atenção primária à saúde bucal no Brasil: processo de trabalho das equipes de saúde bucal. Ciência \& Saúde Coletiva, 24(5), 1809-1820.

Observatório de Análise Política em Saúde. (2021). Implantação. Análise de Políticas de Saúde Bucal no Brasil. https://analisepoliticaemsaude.org/oaps/matriz/analises/1/.

Paiva, R. A., Randow, R., Diniz, L. P., \& Guerra, V. A. (2018). O papel do gestor de serviços de saúde: revisão de literatura. Rev Med, Minas Gerais, (28).

Pereira, C. R. S., Roncalli, A. G., Cangussu, M. C. T., Noro, L. R. A., Patrício, A. A. R., \& Lima, K. C. (2012). Impacto da Estratégia Saúde da Família sobre indicadores de saúde bucal: análise em municípios do Nordeste brasileiro com mais de 100 mil habitantes. Cadernos de Saúde Pública, 28(3), 449-462.

Pereira, J. S. (2013). A humanização na atuação no Programa de Saúde da Família - PSF. Universidade Federal de Minas Gerias. Faculdade de Medicina. Núcleo de Educação em Saúde Coletiva. Trabalho de conclusão de curso. Araçuaí.

Pimentel, F. C., Albuquerque, P. C., Martelli, P. J. L., Souza, W. V., \& Acioli, R. M. L. (2012). Caracterização do processo de trabalho das equipes de saúde bucal em municípios de Pernambuco, Brasil, segundo porte populacional: da articulação comunitária à organização do atendimento clínico. Cadernos de Saúde Pública, 28, 146-157.

Rocha, R. A. C. P., \& Goes, P. S. A. (2008). Comparação do acesso aos serviços de saúde bucal em áreas cobertas e não cobertas pela Estratégia Saúde da Família em Campina Grande, Paraíba, Brasil. Cadernos de Saúde Pública, 24(12), 2871-2880.

Rossi, T. R. A., Chaves, S. C. L., Almeida, A. M. F. L., Santos, C. M. L., \& Santana, S. F. (2018). O financiamento federal da política de saúde bucal no Brasil entre 2003 e 2017. Saúde em Debate, 42 (119), 826-836.

Rossi, T. R. A., Sobrinho, J. E. L., Chaves, S. C. L., \& Martelli, P. J. L. (2019). Crise econômica, austeridade e seus efeitos sobre o financiamento e acesso a serviços públicos e privados de saúde bucal. Ciência \& Saúde Coletiva, 24, 4427-4436.

Silva, Y. C., \& Roquete, F. F. (2013). Competências do gestor em serviços de saúde: análise da produção científica, no período de 2001 a $2011 . ~ R A S, 15,58$.

Simão, J. B., \& Orellano, V. I. F. (2015). Um estudo sobre a distribuição das transferências para o setor de saúde no Brasil. Estudos Econômicos (São Paulo), 45(1), 33-63. 